\title{
Analysis of Spaciotemporal Indicators of Penalty Corners in Field Hockey at the World Cup 2018
}

\author{
Radivoj M. Vasiljev, Valery B. Pokaninov, \\ Irina A. Vasiljeva, Aida R. Hubbatullina \\ and Dmitry L. Korzun* \\ Volga Region State Academy \\ of Physical Culture, Sports and Tourism \\ Kazan, Russian Federation
}

Received 28.01.2021, received in revised form 01.02.2021, accepted 12.02.2021

\begin{abstract}
The article provides a statistical analysis of the data on accounting for penalty corners in field hockey. As in many team sports, one of the decisive moments and a very important playing situation is the penalty corners. Of the total number of goals scored in international competitions, during penalty corners, one third of them lead to a goal.

The article presents the results of the successful delivery of a penalty corner, and its correct performance, which depends on three main components: 1) the player performing the pass from the penalty corner, 2) the player stopping the ball behind the line of the penalty space and 3) the player performing a shot on goal (Fig. 1).

It has been confirmed that the first components are the most stable, although their implementation is equal to or more important than the last phase. It is the shot on goal phase that has a large number of variations, since it can be performed both with a direct shot on goal and with playing on the field.
\end{abstract}

Keywords: field hockey, penalty corner, ball delivery, World Championship, spaciotemporal indicators, methods of "shot from a static position", "shot from a step", "sweep shot from a step".

Research area: physical education.

Citation: Vasiljev, R.M., Pokaninov, V.B., Vasiljeva, I.A., Hubbatullina, A.R., Korzun, D.L. (2021). Analysis of spaciotemporal indicators of penalty corners in field hockey at the World Cup 2018. J. Sib. Fed. Univ. Humanit. Soc. Sci., 14(2), 300-309. DOI: 10.17516/1997-1370-0719.

(C) Siberian Federal University. All rights reserved

* Corresponding author E-mail address: radivojv@gmail.com, valerapokaninov@yandex.ru, 100aida100@rambler.ru, tisa@ mail.ru, korzundl@mail.ru

ORCID: 0000-0002-6563-0199 (Vasiljev); 0000-0002-3349-5244 (Pokaninov); 0000-0003-2640-5818 (Vasiljeva); 00000002-3646-6000 (Hubbatullina); 0000-0002-7825-5615 (Korzun) 


\title{
Анализ пространственно-временных показателей подач штрафного углового в хоккее на траве на чемпионате мира 2018 года
}

\author{
Р.М. Васильев, В.Б. Поканинов, И.А. Васильева, \\ А.Р. Хуббатуллина, Д.Л. Корзун \\ Поволжская государственная академия \\ физической культуры, спорта и туризма \\ Российская Федерация, Казань
}

\begin{abstract}
Аннотация. В статье выполнен статистический анализ данных учета штрафных угловых в хоккее на траве. Как и во многих командных видах спорта, одним из решающих моментов и очень важной игровой ситуацией являются штрафные угловые. От общего количества забитых мячей на международных соревнованиях во время розыгрыша штрафных одна треть из них приводит к голу.

В статье представлены результаты успешности подачи штрафного углового и его правильного технического выполнения, которые зависят от трех основных составляющих: 1) игрока, выполняющего передачу со штрафного углового; 2) игрока, останавливающего мяч за линией штрафного пространства; 3) игрока, выполняющего удар по воротам.

Подтверждено, что первые составляющие являются наиболее стабильными, хотя их выполнение равно или более важно, чем последняя фаза. Именно фаза удара по воротам имеет большое количество вариаций, так как ее можно выполнять как прямым ударом по воротам, так и розыгрышем на поле.
\end{abstract}

Ключевые слова: хоккей на траве, штрафной угловой, подача мяча, чемпионат мира, пространственно-временные показатели, способы подачи «толчком со статического положения», «толчком с шага», «заметающим ударом с шага».

Научная специальность: 13.00.04 - теория и методика физического воспитания.

\section{Введение}

Штрафной угловой в хоккее на траве назначается в ворота соперника при нарушении им правил игры в зоне радиуса либо за грубое нарушение за ее пределами (рис. 1).

Кроме высоких требований к физической подготовке, владению техникой и стратегией ведения игры (Anders and Myers, 2008), спортсмены, выполняющие штрафные угловые, должны обладать психологическими навыками и волевой подготовленностью при выполнении удара, так как на них давит груз ответственности, с которым им необходимо справиться (Fedotova, 2003).

В правилах Международной федерацией по хоккею на траве (FIH 2009) четко прописано, что если первый удар по воротам отличается от толчка из статического положения, толчка с шага или толчка заметающим ударом с шага, тогда мяч должен пересечь линию ворот на высоте не более 460 мм, т. е. на высоте задней доски в воротах (www.thehockeyfamily.com/hockeyterminology; Bari et al., 2014a; Yusoff et al., 2008).

Во время выполнения штрафного углового удар из статического положения, по мнению ряда авторов (McLaughlin, 1997; Piñeiro et al., 2007; Yusoff et al., 2008), намного эффективнее, чем толчок с шага или толчок заметающим ударом с шага. Он используется игроками на всех уровнях хоккея на траве, от люби- 


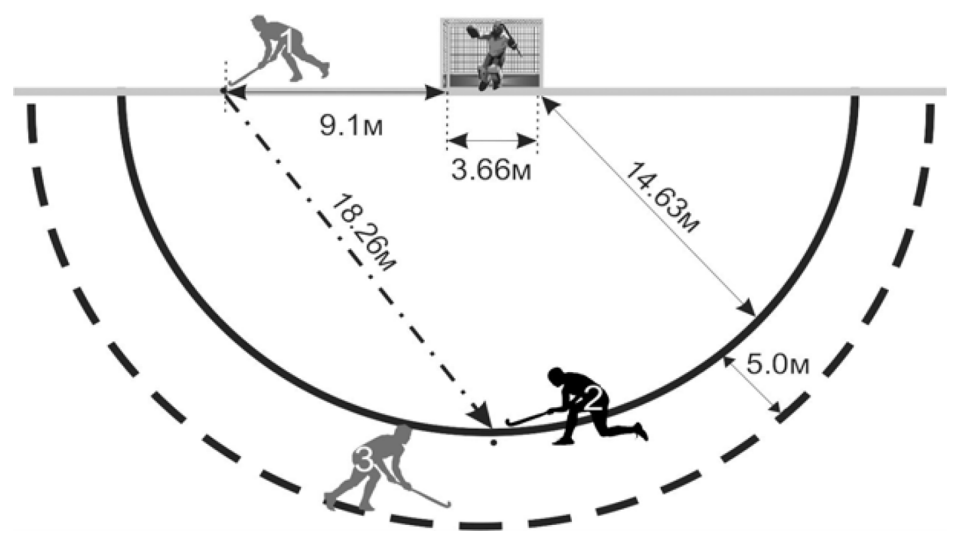

Рис. 1. Расстановка игроков атакующей команды при розыгрыше штрафного углового:

1 - подающий; 2 - принимающий; 3 - забивающий

Fig. 1. Positions of the attacking team players during the penalty corner (1 - injector, 2 - fielder, 3 - scorer)

телей до высшего международного уровня. На соревнованиях штрафной угловой удар из статического положения применяется больше игроками мужского пола, чем женщинами (Piñeiro et al., 2007; Bari et al., 2014b). Подача мяча во время выполнения штрафного углового из статического положения является очень сложной техникой для обучения, требующей координации, силы, ловкости и времени на ее обучение. Она достаточно тяжело тренируется и имеет повышенные требования к организму (Meulman et al., 2012).

Техника выполнения штрафного углового удара разная и отличается по уровню мастерства. Факторами обеспечения высокой эффективности штрафных угловых являются не количество и бесконечное разнообразие вариантов розыгрыша, а надежность и четкость выполнения, как правило, 4-5 вариантов, доведенных до автоматизма. Причем, как показывает практика, разнообразие розыгрышей достигается сегодня не столько за счет передач мяча, сколько за счет отвлекающих и перекрывающих обзор сопернику перемещений игроков без мяча (Gromakov, 2004). Большое значение имеет также быстрота и точность выполнения всех составляющих штрафного углового - подачи, приема мяча и атаки ворот (Fedotova, 2003).
Цель исследования: провести анализ пространственно-временных показателей подачи штрафных угловых в матчах чемпионата мира 2018 года среди ведущих мужских и женских команд.

\section{Методы и организация исследования}

При изучении литературных источников, как российских, так и зарубежных, было установлено, что тема анализа пространственно-временных показателей недостаточно освещена. Проблематику подачи штрафного углового давно не рассматривали. В основном это данные, которые устарели в связи с тем, что поменялись и сами правила игры.

Для выявления таких показателей, как время подачи штрафного углового, точность подачи штрафного углового, вид подачи штрафного углового и реализация данного момента, во время чемпионата мира 2018 года по хоккею на траве использовали видеоанализ с помощью программы в открытом доступе «Kinovea».

Было проанализировано 36 матчей чемпионата мира 2018 года среди женщин в Лондоне (Англия), из них 12 матчей плей-офф и 24 групповых матча. Выделено и проанализировано 248 малых угловых у 16 сборных команд. Также анализировалось 36 матчей чемпионата мира 2018 года 
среди мужчин в г. Бхубанешваре (Индия), из них 12 матчей плей-офф и 24 групповых матча. Из 36 матчей было выделено и проанализировано 245 малых угловых у 16 сборных команд. В общей сложности изучено 72 матча мужских и женских команд.

Регистрировали время подачи, точность подачи, прием подачи и момент реализации.

На основании полученных временных показателей каждого удара при вычисленном нами расстоянии от места подачи подающего игрока к принимающему, которое в среднем составляло 18,24 м (рис. 1), определяли средний показатель скорости перемещения мяча от подающего до принимающего у обеих групп. Время контакта и время приема мяча нами рассмотрены в момент отрыва от клюшки до момента приема игроком.

Статистическая обработка данных проводилась с помощью стандартных статистических методов и с использованием теста * $\varphi$-углового преобразования Фишера (Сидоренко, 2000).

\section{Результаты исследования и их обсуждение}

В таблице 1 даны количественные показатели способа подач у мужчин и женщин и оценка того, насколько точно/неточно они выполнены. Описаны все три вида подачи, которые применялись на турнире: «Толчком со статического положения»; «Толчком с шага» и «Заметающим ударом с шага».

У мужчин заметно преобладание подачи «Толчком со статического положения»
(100\%) и с высокой точностью (98,7\%). $\mathrm{y}$ женщин доминирует подача «Толчком со статического положения» - 68,3 \% (точность 96,2 \%), потом следует подача «Толчком с шага» - 33,1 \% (точность 92,7\%) и «Заметающим ударом с ходу» - 2,5\% (точность 83,3\%). Суммарная точность всех трех разновидностей подачи у женщин составляет 94,3\%.

При изучении выполнения подачи «Толчком со статического положения» обнаружено, что существует статистически значимое отличие между мужчинами и женщинами в проявлении точности подачи (применили * $\varphi$-угловое преобразование Фишера). Оказывается, что мужчины техникой подачи «Толчком со статического положения» более точно попадают и забивают голы, чем женщины $(\mathrm{p} \leq 0,018)$.

Выбор способа подачи у ведущих команд не случаен. «Удар из статического положения» намного эффективнее, чем «толчок с ходу» или «толчок заметающим ударом с ходу» (McLaughlin, 1997; Piñeiro et al., 2007; Yusoff et al., 2008), и при подаче «из статического положения» максимальная скорость последовательной мышечной активации передается на конец клюшки (Ibrahim et al., 2016). Практика показывает, что штрафной угловой удар из статического положения используется больше игроками мужского пола, чем женского (Bari et al., 2014b), что подтвердилось и при анализе матчей ЧМ 2018 года (Vasiljev, 2020). На вопрос, почему это так, можно лишь сделать предположения и предложить несколько вариантов ответов:

Таблица 1. Результативность на ЧМ 2018 года в зависимости от способа подачи Table 1. Performance at the World Championship 2018 depending on the type of delivery

\begin{tabular}{|l|c|c|c|c|}
\hline \multirow{2}{*}{\multicolumn{1}{|c|}{ Способ подачи }} & \multicolumn{2}{|c|}{ Мужчины } & \multicolumn{2}{c|}{ Женщины } \\
\cline { 2 - 5 } & Точно & Не точно & Точно & 6 \\
\hline $\begin{array}{l}\text { Толчком со статического } \\
\text { положения }\end{array}$ & 242 & 2 & 153 & 6 \\
\hline Толчком с шага & отсутствует & отсутствует & 76 & 1 \\
\hline Заметающим ударом с шага & отсутствует & отсутствует & 5 & 13 \\
\hline Суммарно кол-во подач & 242 & 2 & 234 & 5,7 \\
\hline В\% & 98,7 & 1,3 & 94,3 & \\
\hline
\end{tabular}


a) техническая неготовность игроков выполнять четкие и точные подачи (не ведется отдельно тренировочный процесс отшлифовки подачи);

б) физическая неподготовленность опорно-двигательного аппарата к выполнению подачи (нет достаточной массы подающего, чтобы скорость была на требуемом уровне);

в) неосведомленность в преимуществе подачи «Толчком со статического положения» и тактическая неграмотность.

Видеоанализ

пространственновременных показателей у мужчин и женщин при подаче штрафного углового выявил средние показатели времени подачи штрафного углового различными способами у тех и других (табл. 2).

Самым медленным оказывается вид подачи «Толчком со статического положения» (мужчины $1,05 \pm 0,08$ с и женщины 1,27 \pm 0,11 c), а самым быстрым - «Заметающим ударом с шага», регистрированный только у женщин $(0,92 \pm 0,07$ c), которые таким способом делают всего лишь 2,5 \% от всех подач, подают штрафной угловой.

Расчет скорости подачи способом «Толчком со статического положения» у мужчин показал, что она равна 17,37 м/с (62,5 км/ч), у женщин - 14,25 м/с (51,3 км/ч) (табл. 3). Там же обнаружены статистически значимые различия в параметрах скорости перемещения мяча между мужчинами и женщинами при их сравнении в t-тесте с различными дисперсиями для несвязанных выборок $(\mathrm{p}<0,001)$. Скорость подачи у мужчин больше, чем у женщин, они подают более стабильно, и это можно приписать гендерному отличию.

На рис. 2 отображены различия во времени подачи штрафного углового «Толчком со статического положения» у мужчин и женщин при уровне статистической значимости $\mathrm{p}<0,001$. В процентном соотношении время подачи у мужчин меньше на $22 \%$, чем у женщин.

Результаты измерения скорости подач со штрафного углового во время игры в литературе находим противоречивыми. Для наглядного представления результатов различных исследований нами предложена табл. 4, которая оформлена в хронологическом порядке проведения и публикации исследования. Так, показанные значения скорости мяча у мужчин для прицельных подач составляли 11,42 м/с и неприцельных 9,97 м/с (Eskiyecek et. al., 2018). Анализ трех видов подач штрафного углового у испытуемых на примере одного мужчины и одной женщины (dos Santos et al., 2008) показал,

Таблица 2. Время подачи штрафного углового на ЧМ 2018 года

Table 2. Time of delivering penalty corner at the World Championship 2018

\begin{tabular}{|l|c|c|c|c|}
\hline \multirow{2}{*}{\multicolumn{1}{|c}{ Способ подачи }} & \multicolumn{2}{|c|}{ Мужчины, с } & \multicolumn{2}{c|}{ Женщины, с } \\
\cline { 2 - 5 } & Точно & Не точно & Точно & Не точно \\
\hline $\begin{array}{l}\text { Толчком со статического } \\
\text { положения }\end{array}$ & $1,05 \pm 0,08$ & $1,06 \pm 0,14$ & $1,27 \pm 0,11$ & $1,34 \pm 0,16$ \\
\hline Толчком с шага & - & - & $1,2 \pm 0,11$ & $1,5 \pm 0,47$ \\
\hline Заметающим ударом с шага & - & - & $0,92 \pm 0,07$ & 0,9 \\
\hline
\end{tabular}

Таблица 3. Анализ пространственно-временных показателей

Table 3. Analysis of the spaciotemporal indicators

\begin{tabular}{|l|c|c|}
\hline & Мужчины & Женщины \\
\hline Время - t (с) подачи «Толчком со статического положения» & $1,05 \pm 0,08$ & $1,27 \pm 0,11$ \\
\hline v (м/с) перемещение мяча от игрока 1 до игрока 2 & 17,37 & 14,25 \\
\hline Коэффициент вариации (\%) & 7,70 & 9,26 \\
\hline
\end{tabular}




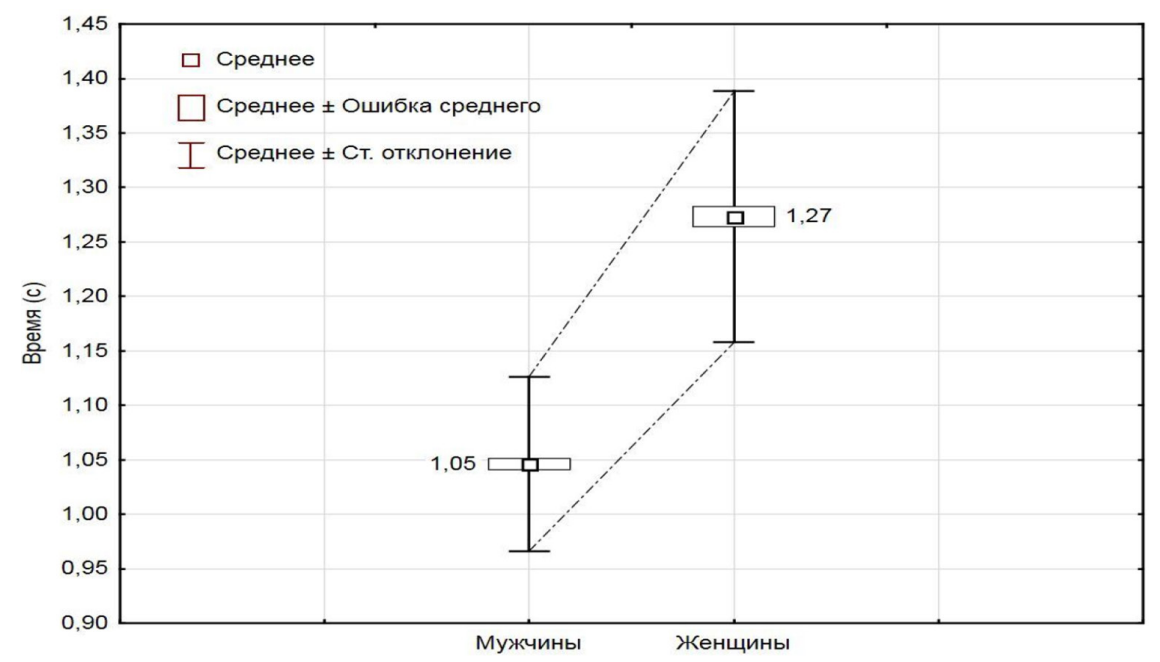

Рис. 2. Время подачи штрафного углового «Толчком со статического положения» у мужчин и женщин

Fig. 2. Time of delivering the penalty corner with the shot from a static positions among men and women

что максимальная скорость мяча на выходе у мужчины (техника «Заметающим ударом с шага») составляет 13,01 м/с, а у женщины при выполнении техникой «Толчком с шага» $-11,4$ м/с.

У элитных испанских игроков-женщин обнаружено, что скорость мяча при подаче с малого углового составляет $18,8 \mathrm{~m} / \mathrm{c}$, у мужчин - 20 м/c (Pérez et al., 2005). При проведенном исследовании у тренированных и нетренированных мужчин-спортсменов (Kerr e Ness, 2006) было обнаружено, что при подаче штрафного углового у тренированных спортсменов скорость мяча составляла 18,01 м/с, а у нетренированных 16,1 м/с. Результаты, которые показали мужчины на ЧМ 2018 года (по нашим расчетам от 17,37 м/с), вполне совпадают с мнением специалистов (McLaughlin's, 1997) о том, что скорость может находиться в диапазоне от 15,2 до $21,9 \mathrm{~m} / \mathrm{c}$. Но некоторые авторы в своих исследованиях (Yussoff et al., 2008) полагают, что скорость находится в диапазоне от 19,6 до 27,8 м/с. Это подтверждают и результаты, полученные в исследовании (López de Subijana et al., 2010), где скорость удара у неопытных игроков мужчин и женщин составила $21,9 \pm 1,7$ и $17,9 \pm 1,7 \mathrm{M} / \mathrm{c}$ соответственно, у игроков мужчин, мастеров международного уровня, 25,4 $\pm 1,3$ м/с. Коридор скоростей, можно отметить, значительно сузился, если его сравнивать с ранее бытующим мнением, что скорость подач находится в широком диапазоне от 10 до $40 \mathrm{~m} / \mathrm{c}$ (Prescott, 1977).

У игроков австралийской мужской сборной при прицельном ударе толчком с поверхности площадки скорость мяча рав-

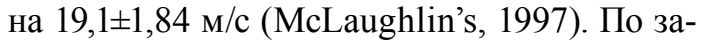
регистрированным результатам при подаче мяча на ЧМ-2018 способ подачи штрафного углового «Толчком со статического положения» как у мужских (17,37 м/с), так и у женских команд $(14,25$ м/с) является «условно модельным» для ведущих мировых команд в хоккее на траве. Учтен тот факт, что результаты получены с помощью видеоанализа реальных соревнований, а не в лабораторных условиях (табл. 4).

Если речь идет о сравнении скорости подачи штрафного углового со скоростью выполнения удара третьего игрока непосредственно при выполнении штрафного удара, бьющего по воротам, тут значения скорости полета мяча больше, если сравнивать ее со скоростью первого игрока, 
Radivoj M. Vasiljev, Valery B. Pokaninov... Analysis of Spaciotemporal Indicators of Penalty Corners in Field Hockey...

Таблица 4. Подача углового удара игроком № 1. Скорость мяча по сведениям различных авторов

Table 4. Penalty corner shot by the player No. 1.

The speed of the ball according to the data by different authors

\begin{tabular}{|c|c|c|c|}
\hline $\begin{array}{l}\text { Авторы (год } \\
\text { публикации) }\end{array}$ & Скорость м/с & Комментарий & Пол (n) \\
\hline Prescott, J.R. (1977) & От 10 до 40 & $\begin{array}{l}\text { Теоретическое предположение } \\
\text { в зависимости от угла выхода мяча }\end{array}$ & - \\
\hline \multirow{2}{*}{$\begin{array}{l}\text { McLaughlin, } \\
\text { P. (1997) }\end{array}$} & От 15,2 до 21,9 & Теоретическое предположение & - \\
\hline & $\begin{array}{l}19,1 \pm 1,84 \\
(\text { от 19,1 до 21,9) }\end{array}$ & Сборная Австралии & Мужчины (14) \\
\hline \multirow[t]{4}{*}{$\begin{array}{l}\text { Pérez, F.J.V. et } \\
\text { al. (2005) }\end{array}$} & $\begin{array}{l}\text { До } 18,47 \pm 0,12 \\
\text { после } 18,69 \pm 1,09\end{array}$ & $\begin{array}{l}\text { В тренировке использовали } 5 \\
\text { различных мячей - результат } \\
\text { со стандартным мячом весом } 160 \text { г }\end{array}$ & Женщины (1) \\
\hline & \begin{tabular}{|l|} 
До 18,62 \\
после 20,0 \\
\end{tabular} & Лучшие подачи до и после тренировки & Женщины (1) \\
\hline & $\begin{array}{l}\text { До } 18,08 \pm 4,26 ; \\
\text { после } 22,05 \pm 0,90\end{array}$ & $\begin{array}{l}\text { До и после в тренировке использовали } \\
5 \text { различных мячей - результат } \\
\text { со стандартным мячом } 160 \text { г }\end{array}$ & Мужчины (1) \\
\hline & $\begin{array}{l}\text { До 22,17; } \\
\text { после 22,62 }\end{array}$ & Лучшие подачи до и после тренировки & Мужчины (1) \\
\hline \multirow{2}{*}{$\begin{array}{l}\text { Kerr, R., Ness, } \\
\text { K. (2006) }\end{array}$} & 18,01 & Тренированные спортсмены & Мужчины (8) \\
\hline & 16,1 & Нетренированные & Мужчины (9) \\
\hline $\begin{array}{l}\text { Yussoff, S. et } \\
\text { al. (2008) }\end{array}$ & От 19,6 до 27,8 & $\begin{array}{l}\text { Предположение, что скорость } \\
\text { может находиться МСМ }\end{array}$ & Мужчины (5) \\
\hline \multirow[t]{2}{*}{$\begin{array}{l}\text { dos Santos, S.G. } \\
\text { et al. (2008) }\end{array}$} & $\begin{array}{l}\text { Техника } \\
\text { № } 1-13,89 \pm 0,46 \\
\text { Техника } \\
\text { № } 2-12,2 \pm 0,43 \\
\text { Техника } \\
\text { № } 3-13,01 \pm 0,70 \\
\end{array}$ & Три различных техники выполнения & Мужчины (1) \\
\hline & $\begin{array}{l}\text { Техника } \\
\text { № } 1-10,93 \pm 1,12 \\
\text { Техника № 2-11,4 } \\
\pm 0,69 \\
\end{array}$ & Две различных техники выполнения & Женщины (3) \\
\hline \multirow{3}{*}{$\begin{array}{l}\text { López de Subijana, } \\
\text { C. et al. (2010) }\end{array}$} & $21,9 \pm 1,7$ & Неопытные игроки & Мужчины (6) \\
\hline & $17,9 \pm 1,7$ & Неопытные игроки & Женщины (6) \\
\hline & $25,4 \pm 1,3$ & Опытные игроки МСМ & Мужчины (1) \\
\hline \multirow[t]{2}{*}{$\begin{array}{l}\text { Eskiyecek, C.G. } \\
\text { et. al (2018) }\end{array}$} & $\begin{array}{l}11,42 \pm 3,58 \text { Мин- } \\
\text { макс 3,06-16,11 } \\
\end{array}$ & Прицельный удар & Мужчины (11) \\
\hline & $\begin{array}{l}9,97 \pm 3,80 \text { Мин- } \\
\text { макс 2,78-14,17 } \\
\end{array}$ & Неприцельный удар & Мужчины (11) \\
\hline Sundar, V. (2019) & $16,49 \pm 0,77$ & $\begin{array}{l}\text { Игроки - студенты } \\
\text { университетов - Малайзия }\end{array}$ & Мужчины (73) \\
\hline
\end{tabular}

подающего мяч. Для наглядности нами предложена табл. 5, в которой отражены результаты величин скорости, полученные различными авторами. В исследовании
(Baker et al., 2009) скорость мяча измерена с помощью радара, она составила $30,5 \mathrm{~m} / \mathrm{c}$. Примерно такую же скорость измерили у студентов колледжа во время бросков 
Таблица 5. Удар по воротам игроком № 3. Скорость мяча по сведениям различных авторов Table 5. Shot on goal by the player No. 3. The speed of the ball according to the data by different authors

\begin{tabular}{|c|c|c|c|c|}
\hline $\begin{array}{l}\text { Авторы (год } \\
\text { публикации) }\end{array}$ & \multicolumn{2}{|c|}{ Скорость м/с } & Комментарий & Пол (n) \\
\hline $\begin{array}{l}\text { Prescott, J.R. } \\
\text { (1977) }\end{array}$ & \multicolumn{2}{|c|}{ до 40} & $\begin{array}{l}\text { Предположение, что скорость может } \\
\text { находиться в этом интервале }\end{array}$ & $\begin{array}{l}\text { Теоретическое } \\
\text { предположение }\end{array}$ \\
\hline $\begin{array}{l}\text { Baker et al. } \\
(2009)\end{array}$ & \multicolumn{2}{|c|}{$\begin{array}{l}\text { 30,5 средняя скорость } \\
\text { подачи drag flick }\end{array}$} & $\begin{array}{l}6 \text { вратарей-профессионалов - } \\
\text { Австралия } \\
10 \text { студентов-новичков - Австралия }\end{array}$ & Мужчины (10) \\
\hline $\begin{array}{l}\text { Hussain et } \\
\text { al. (2011) }\end{array}$ & \multicolumn{2}{|c|}{ от 25,42 до 33,57} & $\begin{array}{l}\text { Предположение, что скорость может } \\
\text { находиться в этом интервале }\end{array}$ & $\begin{array}{l}\text { Теоретическое } \\
\text { предположение }\end{array}$ \\
\hline \multirow{2}{*}{$\begin{array}{l}\text { Hussain et } \\
\text { al. (2012) }\end{array}$} & \multicolumn{2}{|c|}{$31,85 \pm 0,86$} & Клуб «All India Intervarsity», Индия & Мужчины (5) \\
\hline & \multicolumn{2}{|c|}{$30,99 \pm 4,33$} & Клуб «Senior State», Индия & Мужчины (5) \\
\hline \multirow[t]{5}{*}{$\begin{array}{l}\text { López de Subija- } \\
\text { na et al. (2012) }\end{array}$} & Игрок № 1 & $\begin{array}{l}\text { до } 22,5-22,4 \\
\text { после }\end{array}$ & \multirow{4}{*}{$\begin{array}{l}\text { Тренировка до - после, удар drag flick } \\
\text { По воротам с разбега } \\
\text { **стат. значимо изменение } \\
\text { Испания }\end{array}$} & \multirow[t]{4}{*}{ Женщины (4) } \\
\hline & Игрок № 2 & $\begin{array}{c}\text { до } 19,1-18,9 \\
\text { после }\end{array}$ & & \\
\hline & Игрок № 3 & $\begin{array}{c}\text { до } 20,5-21,2^{* *} \\
\text { после }\end{array}$ & & \\
\hline & Игрок № 4 & $\begin{array}{l}\text { до } 19,9-20,0 \\
\text { после }\end{array}$ & & \\
\hline & \multicolumn{2}{|c|}{ От 18,9 до 22,5} & Игроки высшей лиги - Испания & Женщины (4) \\
\hline \multirow{4}{*}{$\begin{array}{l}\text { Bo-Jane Ladru } \\
\text { et al. (2019) }\end{array}$} & \multicolumn{2}{|c|}{$25,62 \pm 2,18$} & Игроки высшей лиги - Нидерланды & Мужчины (12) \\
\hline & \multicolumn{2}{|c|}{$19,50 \pm 2,18$} & Игроки высшей лиги - Нидерланды & Женщины (7) \\
\hline & \multicolumn{2}{|c|}{$25,18 \pm 3,25$} & Взрослые >18 лет - Нидерланды & $\begin{array}{c}\text { Мужчины (9) } \\
\text { и женщины (2) }\end{array}$ \\
\hline & \multicolumn{2}{|c|}{$20,87 \pm 2,08$} & Юниоры <18 лет - Нидерланды & $\begin{array}{c}\text { Мужчины (3) } \\
\text { и женщины (5) }\end{array}$ \\
\hline
\end{tabular}

толчком - 31,85 $\pm 0,86$ м/с и у игроков сборной Индии - 30,99 \pm 4,33 м/c (Hussain et al., 2012).

Под влиянием различных уровней тренировки скорость мяча игроков может находиться в диапазоне от 25,42 до $33,57 \mathrm{~m} / \mathrm{c}$ (Hussain et al., 2011), при этом достигая и $40 \mathrm{~m} / \mathrm{c}$ (Prescott, 1977). Несмотря на то что некоторые исследователи утверждают, что между успешными и неудачными бросками толком нет существенной разницы (Bari et al., 2014b), все-таки результаты исследований показывают, что самая высокая скорость мяча, которая когда-либо была достигнута, внесла важный вклад в «забивание гола» (Bari et al., 2014a).

По сравнению со скоростью мяча от игрока, подающего штрафной угловой, находим, что прямой удар по створу ворот в два раза превышает скорость мяча зарегистрированных нами значений скорости подачи штрафного углового. В соответствии с законом Фитца (Fitt's, 1954) скорость перемещения и точность попадания в цель обратно соотносятся. Чем быстрее движется мяч, тем менее точны удары и тем сам процент попадания уменьшается, и мы показываем, что скорость подачи оптимальна, так как процент хорошего приема большой.

Еще одним фактором, влияющим на точность, является сложность выполнения удара. Другими словами, по мере уменьшения размера цели игроку, выполняющему удар, необходимо дополнительное время на выполнение движения и ве- 
роятность попадания в заданную точку становится меньше. Скорость и точность и в данном случае не коррелируют, и могут потребоваться другие стратегии при выполнении таких двигательных задач. Поэтому можем говорить, что нами зарегистрированные параметры скорости являются на данный момент «условно эталонными» как для мужчин, так и для женщин.

\section{Выводы}

1. Анализ

пространственновременных показателей подач штрафного углового в хоккее на траве на ЧМ-2018 по- казал, что в основном успешно используется способ подачи «Толчком со статического положения».

2. Штрафные подачи «Толчком со статического положения», выполненные мужчинами, более результативные и стабильные, чем у женщин.

3. Время подачи способом «Толчком со статического положения» у мужчин меньше, чем у женщин на $22 \%$.

4. Модельная скорость перемещения мяча от подающего игрока 1 до принимающего игрока 2 у женщин составляет 14,25 и у мужчин - 17,37 м/с.

\section{Список литературы / References}

Anders, E., Myers, S. (2008). Field Hockey: Steps to Success. Champaign: Human Kinetics.

Bari, M.A., Ansari, N.W., Ahmad, F., Hussain, I. (2014a). Three dimensional analysis of dragflick in the field hockey of university players. In Advances in Physics Theories and Applications, 29, 87-93.

Bari, M.A., Ansari, N.W., Hussain, I., Ahmad, F., Ali Khan, M.A. (2014b). Three dimensional analysis of variation between successful and unsuccessful drag flick techniques in field hockey. In International Journal of Research Studies in Science, Engineering and Technology, 2 (1), 74-78.

Dos Santos, S.G., Dal Pupo, J., Piucco, T., Cunha dos Reis, D., Detanico, D. (2008). Análise biomecânica de diferentes técnicas de execução do short corner no hóquei sobre a grama. In Brazilian Journal of Biomotricity, 4 (2), 269-283.

Eskiyecek, C.G. et al. (2018). 3d biomechanical analysis of targeted and non-targeted drag flick shooting technique in field hockey. In Acta Kinesiologica, 2 (12), 13-19.

Fitts, P.M. (1954). The information capacity of the human motor system in controlling the amplitude of movement". In J.Exp. Psychology, (47), 381-391.

Fedotova, E.V. (2003). Osnovy upravlyeniia mnogoletnei podgotovki sportsmenov v komandnykh igrovykh vidakh sporta [Basics of managing the long-term training of athletes in team sports]. Sport Academ Pres, Moscow, 224 p.

Fedotova, E.V. (2009). Azbuka khokeia na trave [ABC of the field hockey]. FHTR, Moscow, 320 p.

Gromakov, V.S. (2004). Podgotovka igroka v khokee na trave [Training the player for field hockey]. In Sport i kul'tura [Sport and Culture]. Moscow, $310 \mathrm{p}$.

Hussain, I., Mohammad, A., Khan, A., Bari, M.A., Ahmad, A., Ahmad, S. (2011). Penalty stroke in field hockey: A biomechanical study. In International Journal of Sports Science and Engineering, 1 (5), 53-57.

Hussain, I., Ahmed, S., Khan, S. (2012). Biomechanical study on drag flick in field hockey. In International Journal of Behavioral Social and Movement Sciences, 3(1), 1-8.

Ibrahim, R., Faber, G.S., Kingma, I., \& Van Dieën, J.H. (2016). Kinematic analysis of the drag flick in field hockey. In Sports Biomechanics, 16 (1), 45-57.

Kerr, R., Ness, K. (2006). Kinematics of de Field Hockey Penalty Corner Push-in. In Sports Biomechanics, 5 (1), 47-61.

López de Subijana, C., Juarez, D., Mallo, J., \& Navarro, E. (2010). Biomechanical analysis of the penalty-corner drag flick of elite male and female hockey players. In Sport Biomechanics, 9, 72-79.

McLaughlin, P. (1997). Three-dimensional biomechanical analysis of the hockey drag flick: Full report, Australia: National Sports Research Centre. (ISBN 064226313 2). 
Meulman, H.N., Berger, M.A., van der Zande, M.E., Kok, P.M., Ottevanger, E.J., \& Crucq, M.B. (2012). Development of a tool for training the drag flick penalty corner in field hockey. In Procedia Engineering, 34, 508-513.

Pérez, F.J.V., Olmo, M.F., Acero, R.M. (2005). Specific strength training of the flick in Field Hockey through over-weighted balls. In Revista Portuguesa de Ciências do Desporto, 1 (5), 40-48.

Piñeiro, R., Sampedro, J., \& Refoye, I. (2007). Differences between international men's and women's teams in the strategic action of the penalty corner in field hockey. In International Journal of Performance Analysis in Sport, 3 (7), 67-83.

Prescott, J.R. (1977). The physics of a controversial goal. In Physics Education, 12, 43-45.

Sidorenko, E.V. (2000). Metody matematicheskoy obrabotki v psikhology [Methods of mathematical processing in psychology]. Saint-Petersburg, Rech', $350 \mathrm{p}$.

The Hockey Family. Available at: www.thehockeyfamily.com/hockey-terminology

Vasiljev, R.M., Hubbatullina, A.R., Pavelkin, I.V., Yakupov, R.A., Vasiljeva, I.A. (2020). Shtrafnye uglovye $\mathrm{v}$ khokee na trave na chempionate mira 2018 goda sredi muzhchin i zhenshchin - analiz [Penalty corners in field hockey at the World Championship 2018 among men and women: Analysis]. In Nauka $i$ sport: sovremennye tendentsii [Science and Sport: Modern Trends], 3 (8), 50-61 DOI: 10.36028/2308-88262020-8-3-50-61

Yusoff, S., Hasan, N., Wilson, B. (2008). Three-dimensional biomechanical analysis of the hockey drag flick performed in competition”. In ISN Bulletin, National Sport Institute of Malaysia, 1, 35-43. 